\title{
Selected aspects related to epidemiology, pathogenesis, immunity, and control of African swine fever
}

\author{
Grzegorz Woźniakowski, Magdalena Frączyk, \\ Krzysztof Niemczuk, Zygmunt Pejsak \\ Department of Swine Diseases, \\ National Veterinary Research Institute, 24-100 Pulawy, Poland \\ grzegorz.wozniakowski@piwet.pulawy.pl
}

Received: December 21, 2015 Accepted: May 9, 2016

\begin{abstract}
African swine fever (ASF) is currently one of the most severe viral infections of domestic pigs, wild boars, and other hosts belonging to Suidae family. ASF is also considered as the most complex and devastating infectious and haemorrhagic disease of swine due to its severe socio-economic impact and transboundary character. ASF it is a notifiable disease and due to the lack of specific treatment and vaccine, the disease can be only limited by the administrative measures comprising wild boar hunting and stamping out of affected pigs. ASF occurred for the first time in Kenya in 1921 while in Europe (Portugal) the virus was detected at the end of the 1950s. In spite of successful eradication of this threat in a number of affected regions, the virus remains endemic in both feral and domestic pigs in Africa and Sardinia. The 'new era' of ASF started in 2007 after its re-introduction to Georgia. Following its intensive expansion, the virus spread to other Caucasian countries, including the territory of the Russian Federation. In 2014 the virus reached Ukraine, Belarus, and, consequently, European Union countries: Lithuania, Latvia, Estonia, and Poland. The occurrence of ASF in wild boars and pigs had a severe impact on both epidemiology and economy because of the national and international transport and trade consequences. Up to date, starting from the February 2014, eighty ASF cases in wild boar and three outbreaks in domestic pigs have been diagnosed. Taking into account the diverse rate of spread in Poland, this review aims to present and discuss the current state of knowledge on ASF including its epidemiology, pathology, transmission, and perspectives of control.
\end{abstract}

Keywords: African swine fever, epidemiology, pathology, transmission, control, review.

\section{Introduction}

African swine fever (ASF) is a contagious haemorrhagic viral disease of domestic pigs, warthogs, wild boars, and other members of Suidae family. The causative agent of the disease is African swine fever virus (ASFV). ASF was diagnosed for the first time in Kenya by Montgomery in 1921. It is believed that ASF was primarily introduced to the territory of Europe (Portugal) in 1957 with the swill from transport ships coming from Angola. Then ASFV spread among the populations of domestic pigs in Portugal and Spain, where it remained for over 30 years. From then on, the virus was reported in many countries of Eastern and Southern Africa. Consequently, ASFV reached new territories of other European countries including France
(1964), Italy (1967, 1969, 1993), Malta (1978), Belgium (1985), and the Netherlands (1986). Apart from Europe, the virus spread to Cuba (1971, 1980), Brazil, the Dominican Republic (1978), and Haiti (1979). The disease was successfully eradicated from all these territories except Sardinia, where the virus has been endemic since 1978 (34). Taking into account the previous experience with ASF in Portugal, Spain and other European countries, a considerable change in dynamics of ASF pathogenesis, epidemiology, and spread is observed $(29,35)$. Since June 2007 , the 'new era' of emerging ASF has begun. Meanwhile, the occurrence of ASFV is no more limited to the subSaharan countries only. After ASFV introduction with pig swill to the Poti docks in Georgia, the virus reached Transcaucasian regions, leading to a considerable 
change in the way of thinking of its epidemiology, but also exerting economic impact on the pig industry. The virus was rapidly transferred to Armenia, Chechnya, Azerbaijan, the Russian Federation (RF) (2007), and Iran (2009). Subsequently, ASFV expanded its geographical distribution to other European countries including Ukraine (2012) and Belarus (2013). Then, in February 2014, ASFV re-emerged in the European Union (EU), spreading to the territories of Latvia, Poland, Lithuania, and Estonia $(32,35)$.

Epidemiology of ASF. ASFV is infectious to different members of Suidae family including: domestic pigs (Sus scrofa $f$ : domesticus), European wild boars (Sus scrofa scrofa), warthogs (Phacochoerus aethiopicus), bush pigs (Potamochoerus porcus), and giant forest hogs (Hylochoerus meinertzhageni). Within Africa, the natural hosts of ASFV are warthogs and bush pigs, which act as a reservoir of the pathogen. ASFV is also capable of replication in ticks belonging to Ornithodoros genus. The virus may be transmitted by transovarial, transtadial, and sexual routes $(7,35,36)$. While ASFV transmission in Ornithodoros moubata and Ornithodoros porcinus present in Africa can be transovarial, transtadial, or sexual, the transmission in Ornithodoros erraticus occurring in the Iberian Peninsula region can be transtadial and transovarian only. The last report indicates that European ASFV Georgia 2007/1 isolate replicates in Ornithodoros erraticus ticks only $(9,36)$. Other ticks, Ixodes ricinus and Dermacentor reticulatus, are common arthropods (mites) in Europe and are involved as a vector in transmission of many infectious agents: Babesia canis, Theileria equi, Rickettsia spp., or tick-borne encephalitis virus (TBEV) (18). The role of these ticks in ASF epidemiology was investigated (8). However, the results clearly showed that, under in vitro conditions, Ixodes ricinus and Dermacentor reticulatus are not able to transmit ASFV. Two main transmission cycles are established in ASF epidemiology: 'sylvatic' and 'domestic' (7). Both cycles refer to maintenance and transmission of ASFV depending on the environmental and geographical conditions of local area. The sylvatic cycle (wild cycle) is attributed to the most of South and East African countries, where ASFV is present in population of wild suids and soft ticks from Ornithodoros genus, with occasional spread of the virus to domestic pigs. The term 'domestic cycle' refers to wild boar and domestic pig herds where ASFV circulates mainly due to the efficient direct or indirect transmission (16). According to the most recent data, the involvement of tick-vector in Europe is restricted only to the Iberian Peninsula (4). As previously mentioned, the most important for the spread of ASFV is the direct transmission due to close contact between the infected and susceptible animals by ingesting or inhaling the virus from the remains or secretions present in the environment. The virus from infected animals can be transmitted indirectly, involving ticks as a vector, or by biting flies, people, livestock vehicles, fomites, or contaminated food products. Based on reports by Penrith and Vosloo (29), the water birds or rodents are not a potential source of infection. In spite of ASFV presence in genital secretions, there is no evidence for horizontal transmission of the infection during pregnancy in pigs (29).

ASFV molecular structure and features. ASFV is the only member of the Asfivirus genus within Asfarviridae family. Primarily, it has been classified into the Iridoviridae family due to icosahedral morphology of a large virion and the cytoplasmic location of its replication. ASFV virion shares some similarities with Poxviridae viruses including the genomic structure (terminal cross-links and inverted terminal repeats) as well as the replication mechanism and cytoplasmatic localisation. Similarly to other poxviruses, ASFV encodes the enzymes necessary for its replication and transcription $(15,38)$. Consequently, the International Committee on Taxonomy of Viruses (ICTV), during the meeting in 1999, decided to move it from Iridoviridae to the newly created Asfarviridae family (11). The ASFV virion consists of a nucleoprotein core, also called matrix (viral DNA surrounded by a thin protein envelope-core shell). This nucleoprotein core has two lipid membranes comprised of inner lipid membrane which separates nucleoid from the icosahedral-shaped protein capsid, and an external lipid envelope (Fig.1). The latter is an additional layer that is acquired by budding from the host cell membrane (31). The ASFV capsid, which is icosahedral in shape, contains 1892-2172 hexagonal capsomers with 13-nm in length and $5 \mathrm{~nm}$ in width (5, 31 ). As it was previously mentioned, the virus core contains the enzymes required for early mRNA synthesis and processing. The final size of the virion is between 70 and $120 \mathrm{~nm}$ in diameter. The ASFV genome is cross-linked at its ends by two 37 nucleotide-long hairpin loops and inverted terminal repeats (ITRs) ranging from 2.1 to 2.5 kilo base pairs (kbp) in length $(15,38,40)$. The ASFV genetic material is represented by a linear double-stranded DNA with the size ranging from 170 to $190 \mathrm{kbp}$, dependent on the virus genotype or isolate. The DNA structure consists of central conserved region (CCR) which is about $125 \mathrm{kbp}$ long and two flanking variable ends (left-LVR and right- RVR) (2). The CCR region can be further characterised by a short central variable region (CVR) (40). The genetic differences within CVR are related to the number and type of tandemly repeated amino acid tetramers. Left variable 5'-end (LVR) has between 38 and $47 \mathrm{kbp}$ while the RVR 3'-end between 13 and $16 \mathrm{kbp}$ in length, respectively (2). The regions close to the ends of ASFV genome are variable and responsible for the diversity of the virus strains. This is mainly due to multiple deletions and additions. The LVR and RVR encode multigene family genes (MGFs) including MGF100, MGF110, MGF300, MGF360, and MGF505/530 (40). It has been found that ASFV genome contains 151-167 open reading frames - ORFs (11). 


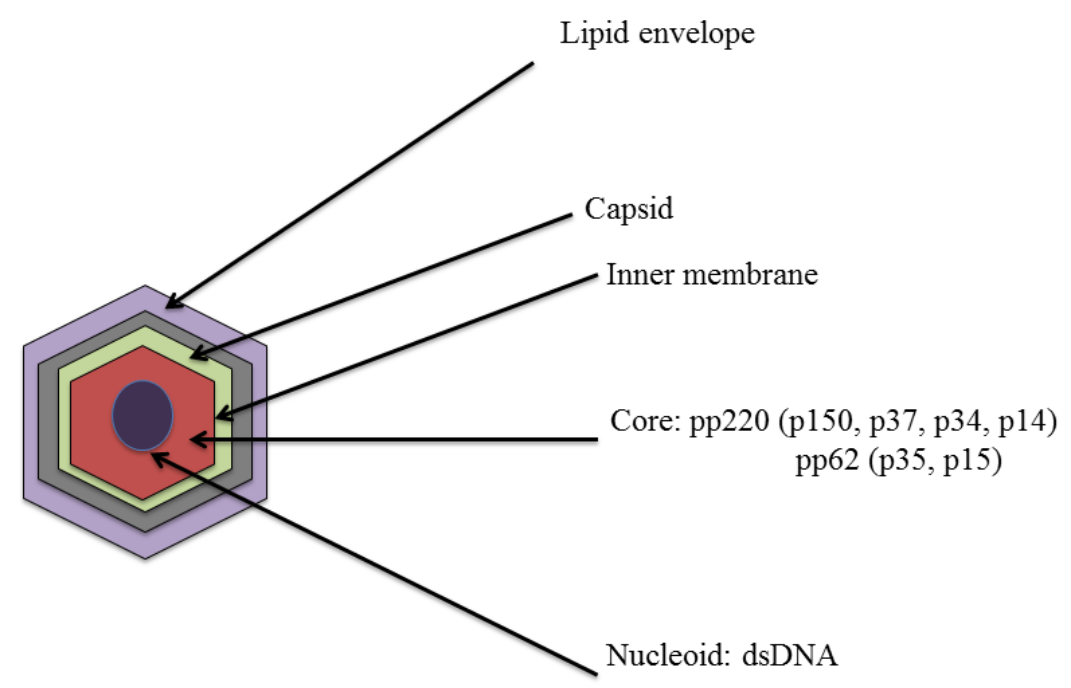

Fig. 1. The molecular structure of African swine fever virus (ASFV). The ASFV virion consists of a core proteins pp220 complex (p150, p37, p34, and p14) and pp62 complex (p35, p15). ASFV also has two lipid membranes comprising inner lipid membrane and external lipid envelope. The ASFV genetic material is represented by a linear double-stranded DNA with the size ranging from 170 to $190 \mathrm{kbp}$

The particular ORFs encode different proteins including structural proteins, enzymes required for ASFV replication, and regulatory proteins involved in immunological interactions with the host cell $(10,39$, 40). The virion is assembled from 54 types of structural proteins form the pool of all encoded proteins. The major component of the virion is $\mathrm{p} 73$ capsid protein encoded by the B646L gene, which comprises $30 \%$ of the total virion molecular mass. The p72 protein of ASFV plays a major role in immunological response. The protein contains a 26 amino acid region which is recognised by cytotoxic T-lymphocytes (21).

The ASFV isolates can be grouped into 22 genotypes according to their nucleotide sequence of the B646L gene sequence which encodes C-terminal end of p72 major capsid protein. This ASFV genomic region is highly conserved $(1,23)$. The analysis of additional genes is required for further differentiation within genotypes and identification of possible subgroups. Further analysis can be done by sequencing E183-L gene encoding p54 envelope protein or $\mathrm{B} 602 \mathrm{~L}$ gene within CVR (12). This molecular typing is helpful to trace the virus spread and replication dynamics due to the dependence between disease outbreak and the occurrence of different viral subtypes within the geographical regions. The occurrence of all 22 genotypes has been described in the African continent, precisely within its eastern and southern parts. The particular genotypes including V, VI, IX, XI, XIII, $\mathrm{XIV}, \mathrm{XV}$, and XVI are assigned to the country. In contrast, genotypes I, II, VIII, X, and XII spread worldwide (23). The outbreaks among domestic pigs and wild boars in Europe were caused predominantly by the Georgia 2007/1 isolate which belongs to the genotype II, primarily originating from Mozambique and Zambia, which also reached the Indian Ocean islands of Madagascar and Mauritius (12, 32). The genotype I is linked to the western and central parts of Africa, but it was also introduced into Europe and South America (7). Currently, the ASFV isolates detected outside Africa belong exclusively to genotypes I and II.

Pathogenesis. The ASFV host range is restricted to Suidae family members and there is no evidence of the impact of the virus on human health. The literature records refer to the possible presence of ASFV-like genome sequences in human sera and sewage in Spain. These data also indicate the similarity of these genetic fragments to asfarviruses, though they are still highly divergent from the ASFV genomic sequences (22). ASFV infects and replicates in cytoplasm of the reticuloendothelial or mononuclear phagocytic system, mainly in monocytes and macrophages, which seems to be crucial for pathogenesis $(3,30)$. In spite of primary monocytes and macrophages, during the further stages of infection ASFV also infects neutrophils, megakaryocytes, endothelial cells, or hepatocytes. Despite significant lymphopenia caused by damage and virus-induced apoptosis, $\mathrm{T}$ and $\mathrm{B}$ lymphocytes are not the target for viral replication. In fact, apoptosis is due to the presence of cytokines released from infected macrophages (30). After adsorption of ASFV to the surface protein receptor, the virus enters the host cell. The natural route of ASFV infection is the alimentary tract. However, other routes, such as the respiratory tract, skin injuries, injections, and tick bites, have also been described $(29,34)$. Independently from the route of infection, the virus spreads via blood stream and lymph, thus the viraemia usually begins $8 \mathrm{~h}$ post infection in the tonsils and lymph nodes. After 15-24 h post infection, the virus spreads via blood stream and lymph from the primary sites of replication to almost 
all tissues of the body. The principal tissues and organs of secondary ASFV propagation include the spleen, bone marrow, liver, lungs, kidneys, and endothelium of organs associated with mononuclear phagocytic system (3). The clinical signs and lesions observed in pigs, including high fever, haemorrhages in skin, or neurological disorders, may be confused with other pig diseases (e.g. classical swine fever, porcine reproductive and respiratory syndrome, swine erysipelas, porcine dermatitis and nephropathy syndrome, septicaemic salmonellosis). In the case of ASF, the incubation period may range from 4 to 19 days after exposure (34) or 18 and $24 \mathrm{~h}$ after experimental intramuscular injection (19). Depending on the route and infection progress as well as the virulence of particular strain, at least a few clinical pictures of ASF are distinguished. The clinical form of the disease might be peracute, acute, subacute, chronic, or subclinical $(3,35)$. Acute to peracute forms may develop after infection of pigs with highly virulent ASFV strains. These two forms are characterised by a high morbidity and mortality up to $100 \%$. The chronic and subacute forms occur when the virus is circulating within the population of pigs or wild boars, thus its virulence is also reduced. During peracute form the pigs die within 1-4 days post infection, without any clinical signs. The only clinical signs that might be observed is fever or apathy. The acute ASF is characterised by typical clinical signs caused by the strong affinity of ASFV to the host vascular system. The initial signs include high body temperature $\left(\sim 42^{\circ} \mathrm{C}\right)$, anorexia, depression, reddened skin of ears, abdomen, and chest with cyanosis, conjunctivitis, and nasal discharge. Subsequently, additional signs may occur including: dyspnea, oedema, vomiting, diarrhoea (often bloody), incoordination and convulsions. The affected animal dies within 3 to 8 days after infection (35). Post-mortem examination frequently reveals ASF specific lesions and petechiae in internal organs such as lymph nodes, kidneys, heart, and bladder, splenomegaly, and hemorrhages in pleura or in the dura mater of the brain $(19,35)$. Subacute picture of the infection is induced by moderately virulent strains. The observed signs or lesions are less pronounced in comparison to the acute form. The observed fever in pigs is lower. The spleen may be enlarged but without discoloration, and haemorrhages in the kidneys are more visible. The subacute form leads to death within 15-45 days or to recovery after 30-45 days. Chronic form of ASF may be manifested by non-specific clinical signs including recurrent fever and emaciation. The necrotic skin lesions, arthritis, or abortions in sows may also be observed. This form of the disease is caused by low virulent ASFV strains and may be detected in carrier animals $(34,35)$.

Immunity. The immune response after ASFV infection has not been completely explained. ASFV has its own mechanisms to manipulate the host immune response and to modulate cell functions leading to recognition, prevention, and inhibition of cell death (6). The virus replicates in mononuclear-phagocytic cells, and thus may cause disorders in releasing proinflammatory cytokines as well as coagulopathy disorders (3). Additionally, ASFV enables mechanisms to evade detection and dysregulation of innate immune responses. One of these mechanisms is manipulation of the signalling pathway related to infected macrophages and expression of genes involved in the innate and acquired immunity $(6,10)$. This results in inhibition of lymphocytes activation and inflammatory response to ASFV. In regard to the humoral immune response, some authors present an opinion that after ASFV infection the production of neutralising antibodies is not induced (17). On the other hand, the reports from other studies showed that the neutralising antibodies are produced against p30, p54, and p72 proteins (13). In fact, ASFV-specific antibodies do not completely neutralise the virus and are not able to protect against infection $(25,33)$. Production of specific ASFV antibodies may provide some protection which could influence the clinical course of ASF, including reduction of viraemia or delay of the clinical signs onset. However, this was demonstrated by the passive transfer of anti-ASFV immunoglobulins to experimentally infected pigs (26). Indeed, Schlafer et al. (37) showed the protective properties of immunoglobulins passively transferred to suckling piglets via colostrum from their convalescent mothers. The susceptibility to neutralisation is determined by the presence of phosphatidylinositol in viral envelope and can be reduced by multiple passaging in vitro (14). Concluding the humoral response against ASFV, the specific antibodies persist for a long time but do not neutralise the virus. The specific antibodies may elicit protection against homologous strain but not heterologous. It is also clear that cellular response against ASFV plays a major role in control of the progress of infection (39). This was experimentally confirmed in ASFV-infected pigs with ASFV-specific cytotoxic $\mathrm{T}$ lymphocyte (CTL) activity destroying infected macrophages (24). There is also evidence for an important role of natural killer cells (NK) in cellular response against ASFV $(20,24)$. Lower NK activity was indeed reported in pigs showing acute clinical picture. The lysis of infected cells was shown to be mediated by $\mathrm{CD} 8+\mathrm{T}$-lymphocytes. It was also demonstrated that depletion of $\mathrm{CD} 8+$ lymphocytes resulted in the loss of protection against ASFV (27).

ASF control measures and diagnosis. Following the lack of specific treatment or vaccine against ASF, the disease can be only controlled by the administrative measures of EU legislation and governmental regulations (41). In Poland, as in other EU countries, ASF is limited by reducing wild boar population as well as stamping out pigs from the backyard holding within the restricted area due to the occurrence of this disease. 


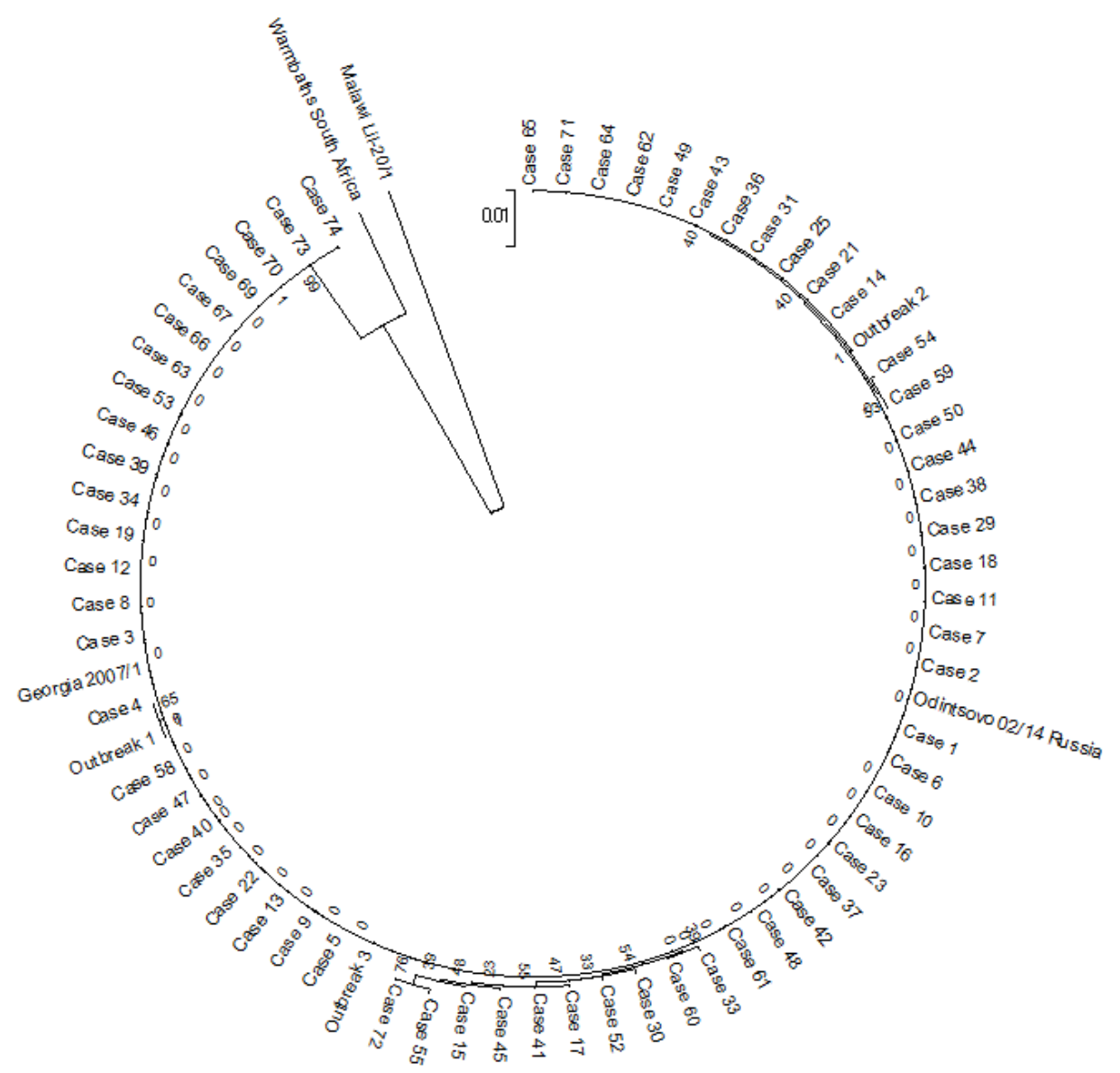

Fig. 2. Phylogenetic tree constructed on the basis of the aligned sequences of MGF-505-2R gene derived from 67 ASFV field isolates and 5 reference strains as Georgia 2007/1 and Odintsovo 2/2014 (Genotype II), Ken06.Bus (Genotype IX), Ba71V and NHV (Genotype I). The tree shows the relationships between particular genotypes and field ASFV isolates circulating in Poland. The scale bar indicates the number of nucleotide substitutions per residue

The other important factors include implementation of hygienic and sanitary requirements as well as restrictions associated with pig or pig meat product movement as well as quarantine and compartmentalisation of the areas. The other important aspect influencing the biosecurity is associated with accurate laboratory diagnosis. Because of the contagious character of ASFV as well as the economical importance of its occurrence, the diagnostic studies on this pathogen are mainly conducted by the National Reference Laboratories (NRLs) authorised by Chief Veterinary Officers of particular countries. The NRL for ASF at the National Veterinary Research Institute in Pulawy (NVRI) performs diagnosis of ASF using OIE and European Reference Laboratory (EURL) recommended diagnostic methods. All diagnostic techniques recommended for ASF diagnosis have been fully described and reviewed in details (28). Diagnostic methods used within NRL in Pulawy are represented by real-time PCR with Universal Probe Library (UPL), enzyme-linked immunosorbent assay (ELISA) for screening of ASFV antibody and confirmatory tests including immunoperoxidase test (IPT) and immunoblot assay (IB). However, it should be taken into account that ASFV DNA may be detected during early and acute stage of wild boar or pig infection. The ASFV DNA is also detected in wild boar remains and bones. On the other hand, no specific antibodies can be detected by ELISA or IPT during viraemia since the antibodies are produced 7-10 days after infection. Therefore, an appropriate diagnostic method should be applied for detection of possible ASFV infection and relevant interpretation of the obtained results is required to draw the final conclusion.

Current situation in Poland. ASF is still one of the major challenges for the Polish State Veterinary Services. The virus has been circulating in wild boar population since the beginning of 2014. Until $22^{\text {nd }}$ of December 2015, 81 ASF cases in wild boar and three outbreaks in swine were confirmed. In that period more than 10,200 samples from hunted or dead wild boars and 13,600 samples from pigs were tested at the NVRI with real-time PCR, ELISA, and/or IPT. Passive surveillance (testing dead wild boars) revealed the annual prevalence of approximately $14 \%$, whereas active surveillance (hunted wild boar) showed a small detection rate with the annual prevalence of $0.12 \%$. All ASF cases and outbreaks were detected in the vicinity of the Belarussian border in the Podlaskie Province and not a single case was detected outside the restricted 
area. Slow spread of ASF contradicts initial assumptions of rapid progress of the disease. Instead of continuous infections in wild boars, a new stage of pathogenesis seems to enter, as indicated by recent detection of several wild boars which survived long enough to clear the infection and develop humoral response. The recent findings from the phylogenetic study on the basis of MGF505-2R variable fragment showed that all Polish isolates fall into the common phylogenetic group with other strains belonging to the genotype II from the territory of Georgia and Russia (Fig.2). However, further studies are required in order to fully understand the phylogenetic relationships of circulating ASFV isolates.

In conclusion, the reviewed aspects associated with epidemiology, pathology, disease progress, and immune response to ASFV infection are extremely important taking into account different socioeconomical aspects of the occurrence of the disease. In spite of a multiplicity of different administrative measures associated with ASF occurrence, the disease presents a major and currently one of the most important threats in production of pigs, as in Polish climate zone ASFV is mainly transmitted by wild boars and their migration. Further analyses of the usefulness of diagnostic methods and interpretation of obtained results are required to understand the aspects of the 'new era' of ASF in Eastern Europe, including Poland.

Conflict of Interests Statement: The authors have no conflict of interests regarding the publication of this article.

\section{References}

1. Bastos A.D., Penright M.L., Cricière C., Edrich J.L., Hutchings G., Roger F., Couacy-Hymann E.R., Thomson G.: Genotyping field isolates of African swine fever virus by partial p72 gene characterisation. Arch Virol 2003, 148, 693-706.

2. Blasco R., Aguero M., Almendral J.M., Vinuela E.: Variable and constant regions in African swine fever virus DNA. Virology 1989, 168, 330-338.

3. Blome S., Gabriel C., Beer M.: Pathogenesis of African swine fever in domestic pigs and European wild boar. Virus Res 2013, 173, 122-130

4. Boinas F.S., Wilson A.J., Hutchings G.H., Martins C., Dixon L.J.: The persistence of African swine fever virus in fieldinfected Ornithodoros erraticus during the ASF endemic period in Portugal. PLoS ONE 2011, 6, e20383.

5. Carrascosa J.L., Carazo J.M., Carrascosa A.L., Garcia N., Santisteban A., Viñuela E.: General morphology and capsid fine structure of African swine fever virus particles. Virology 1984, $132,160-172$

6. Correia S., Ventura S., Parkhouse R.M.: Identification and utility of innate immune system evasion mechanisms of ASFV. Virus Res 2013, 173, 87-100.

7. Costard S., Mur L., Lubroth J., Sanchez-Vizcaino J.M., Pfeiffer D.U.: Epidemiology of African swine fever virus. Virus Res 2013, 173, 191-197.

8. De Carvalho Ferreira H.C, Zúquete S.T., Wijnveld M., Weesendorp E., Jongejan F., Stegeman A., Loeffen W.L.: No evidence of African swine fever virus replication in hard ticks. Ticks Tick-borne Dis 2014, 5, 582-589
9. Diaz A.V., Netherton C.L., Dixon L.K., Wilson A.J.: African swine fever virus strain Georgia 2007/1 in Ornithodoros erraticus ticks. Emerg Infect Dis 2012, 18, 1026-1028.

10. Dixon L.K., Abrams C.C., Bowick G., Goatley L.C., Kay-Jackson P.C., Chapman D., Liverani E., Nix R., Silk R., Zhang F.: African swine fever virus proteins involved in evading host defence systems." Vet Immunol Immunopathol 2004, 100, 117-134.

11. Dixon L.K., Escribano J.M., Martins C., Rock D.L., Salas M.L., Wilkinson P.J. In: Virus Taxonomy. VIII. Report of the ICTV, edited by Fauquet C.M., Mayo M.A., Maniloff J., Desselberger U., Ball L.A., Elsevier Academic Press, London 2005, pp. 135-143.

12. Gallardo C., Mwaengo D.M., Macharia J.M., Arias M., Taracha E.A., Soler A., Okoth E., Martin E., Kasiti J., Bishop R.P.: Enhanced discrimination of African swine fever virus isolates through nucleotide sequencing of the p54, p72, and pB602L (CVR) genes. Virus Genes 2009, 38, 85-95.

13. Gomez-Puertas P., Rodriguez F., Oviedo J.M., RamiroIbanez F., Ruiz-Gonzalvo F., Alonso C., Escribano J.M.: Neutralizing antibodies to different proteins of African swine fever virus inhibit both virus attachment and internalization. J Virol 1996, 70, 5689-5694.

14. Gomez-Puertas P., Oviedo J.M., Rodriguez F., Coll J., Escribano J.M.: Neutralization susceptibility of African swine fever virus is dependent on the phospholipid composition of viral particles. Virology 1997, 228, 180-189.

15. Gonzales A., Talavera A., Almendral J.M., Vinuela E.: Hairpin loop structure of African swine fever virus-DNA. Nucleic Acids Res 1986, 14, 6835-6844.

16. Guinat C., Gogin A., Blome S., Keil G., Pollin R., Pfeiffer D.U., Dixon L.: Transmission routes of African swine fever virus to domestic pigs: current knowledge and future research directions. Vet Rec 2016, 178, 262-267.

17. Hess W.R.: African swine fever: a reassessment. Adv Vet Sci Comp Med 1981, 25, 39-69.

18. Hubálek Z., Rudolf I.: Tick-borne viruses in Europe. Parasitol Res 2012, 111, 9-36.

19. Karalova E., Zakaryan H., Voskanyan H., Arzumanyan H., Hakobyan A., Nersisyan N., Saroyan D., Karalyan N., Tatoyan M., Akopian J., Gazaryantz M., Mkrtchyan Z., Pogosyan 1., Nersesova L., Karalyan Z.: Clinical and postmortem investigations of genotype II induced African swine fever. Porcine Res 2015, 5, 1-11.

20. Leitao A., Cartaxeiro C., Coelho R., Cruz B., Parkhouse R.M., Portugal F., Vigario J.D., Martins C.L.: The non-haemadsorbing African swine fever virus isolate ASFV/NH/P68 provides a model for defining the protective anti-virus immune response. J Gen Virol 2001, 82, 513-523.

21. Leitao A., Mulau A., Cornelis P., Martins C.L.V.: Identification of a 25-amino acid sequence from the major African swine fever virus structural protein VP72 recognised by porcine cytotoxic T lymphocytes using a lipoprotein based expression system. J Virol Methods 1998, 75, 113-119.

22. Loh J., Guoyan Z., Presti R.,M., Holtz L.R., Finkbeiner S. R., Droit L., Villasana Z., Todd C., Pipas J.L., Calgua B., Girones R., Wang D., Virgin H. W.: Detection of novel sequence related to African swine fever virus in human serum and sewage. J Virol 2009, 83, 13019-13025.

23. Lubisi B.A., Bastos A.D., Dwarka R.M., Vosloo W.: Molecular epidemiology of African swine fever in East Africa. Arch Virol 2005, 150, 2439-2452.

24. Martins C.L.V., Leitao A.C.: Porcine immune responses to African swine fever virus (ASFV) infection. Vet Immunol Immunopathol 1994, 43, 99-106.

25. Neilan J.G., Zsak L., Lu Z., Burrage T.G., Kutish G.F., Rock D.L.: Neutralizing antibodies to African swine fever virus proteins $\mathrm{p} 30, \mathrm{p} 54$, and $\mathrm{p} 72$ are not sufficient for antibodymediated protection. Virology 2004, 319, 337-342.

26. Onisk D.V., Borca M.V., Kutish G., Kramer E., Irusta P., Rock D.L.: Passively transferred African swine fever virus 
antibodies protect swine against lethal infection. Virology 1994, 198, 350-354.

27. Oura C.A., Denyer M.S., Takamatsu H., Parkhouse R.M.: In vivo depletion of CD8+ T lymphocytes abrogates protective immunity to African swine fever virus. J Gen Virol 2005, 86, 2445-2450.

28. Oura C.A.L., Edwards L., Batten C.A.: Virological diagnosis of African swine fever - Comparative study of available tests. Virus Res 2013, 173, 150-158.

29. Penrith M.L., Vosloo W.: Review of African swine fever: transmission, spread and control. J S Afr Vet Assoc 2009, 80, 58-62.

30. Ramiro-Ibanez F., Ortega A., Brun A., Escribano J.M., Alonso C.: Apoptosis: a mechanism of cell killing and lymphoid organ impairment during acute African swine fever virus infection. J Gen Virol 1996, 77, 2209-2219.

31. Rouillier I., Brookes S.M., Hyatt A.D., Windsor M., Wileman T.: African swine fever virus is wrapped by the endoplasmic reticulum. J Virol 1998, 72, 2373-2387.

32. Rowlands R.J., Michaud V., Heath L., Hutchings G., Oura C., Vosloo W., Dwarka R., Onashvili T., Albina E., Dixon L.K.: African swine fever virus isolate Georgia 2007. Emerg Infect Dis 2008, 14, 1870-1874.

33. Ruiz Gonzalevo F., Carnero M.E., Caballero C., Martinez J.: Inhibition of African swine fever infection in the presence of immune sera in vivo and in vitro. Am J Vet Res 1986, 47, 1249-1252.
34. Sánchez-Vizcaíno J.M., Arias M.: African swine fever. In: Diseases of swine. Edited by Zimmerman J., Karriker L., Ramirez A., Schwartz K., Stevenson G., Blackwell Publishing Professional, Ames, Iowa 2012, pp. 396-404.

35. Sánchez-Vizcaíno J.M., Mur L., Gomez-Villamandos J.C., Carrasco L.: An update on the epidemiology and pathology of African swine fever. J Comp Path 2015, 152, 9-21.

36. Sánchez-Vizcaíno J.M., Mur L., Bastos A.D., Penrith M.L.: New insights into the role of ticks in African swine fever epidemiology. Rev Sci Tech 2015, 34, 503-511.

37. Schlafer D.H., Mebus C.A., McVicar J.W.: African swine fever in neonatal pigs - passively acquired protection from colostrum or serum of recovered pigs. Am J Vet Res 1984, 45, 1367-1372.

38. Sogo J.M., Almendral J.M., Talavera A., Vinuela E.: Terminal and internal inverted repetitions in African swine fever virus DNA. Virology 1984, 133, 271-275.

39. Takamatsu H.H., Denyer M.S., Lacasta A., Stirling C.M., Argilaguet J.M., Netherton C.L., Oura C.A., Martins C., Rodriguez F.: Cellular immunity in ASFV responses. Virus Res 2013, 173, 110-121.

40. Yanez R.J., Rodriguez J.M., Nogal M.L., Yuste L., Enriquez C., Rodriguez J.F., Vinuela E.: Analysis of the complete nucleotide sequence of African swine fever virus. Virology 1995, 208, 249-278.

41. Zakaryan H., Revilla Y.: African swine fever virus: current state and future perspectives in vaccine and antiviral research. Vet Microbiol 2016, 185, 15-19. 